\title{
Measuring boron nitride films from thick to thin
}

\section{Appl. Phys. Lett. 116, 081104 (2020)}

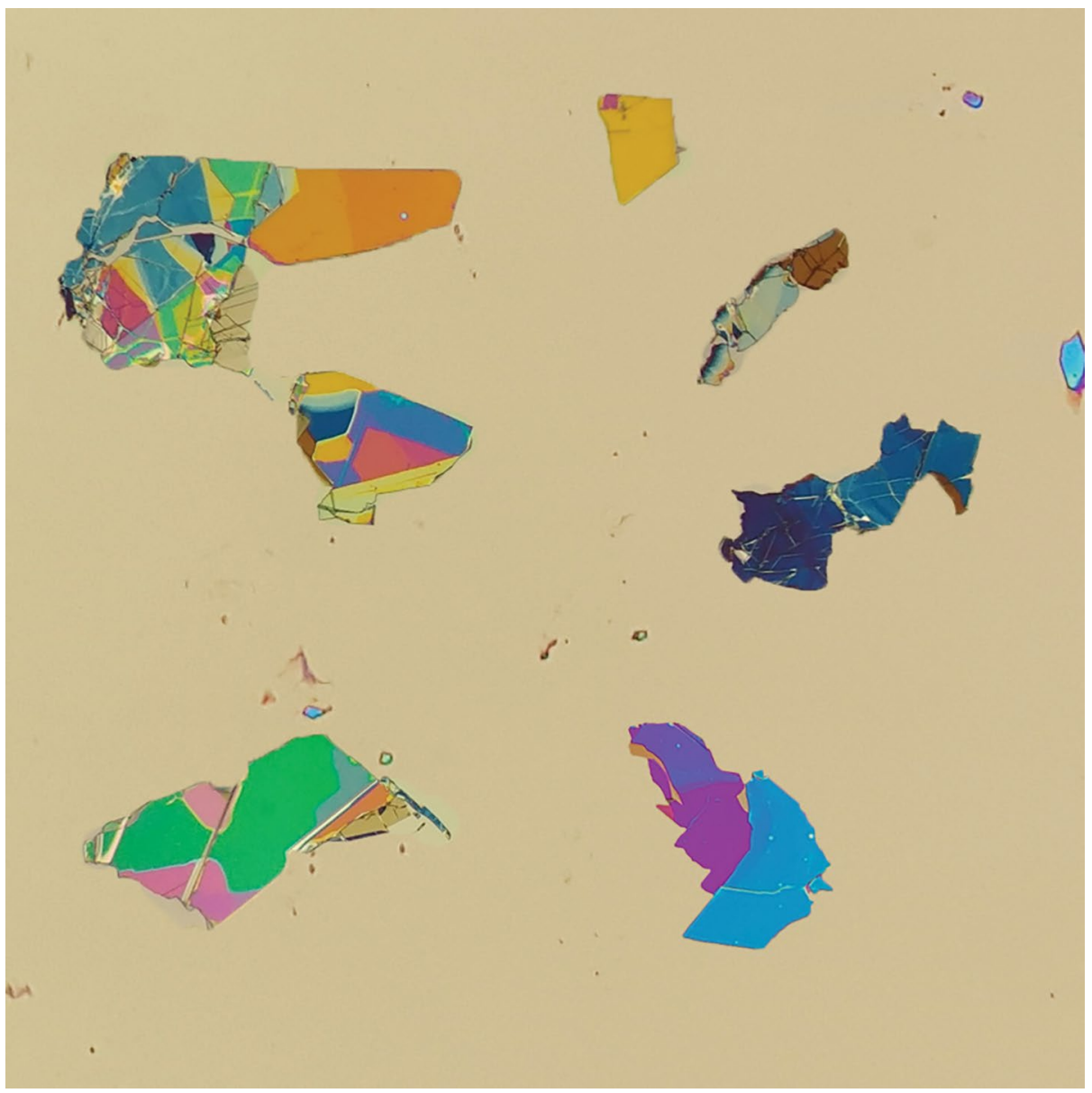

Credit: AIP

Hexagonal boron nitride $(\mathrm{hBN})$ is a twodimensional material with a large bandgap and is used in a variety of electronic devices, including as an ultrathin gate dielectric in transistors and as a host material in tuneable photonic crystal cavities. These different applications require layered hBN films with thicknesses from a few nanometres to hundreds of nanometres, but it is difficult to accurately and quickly characterize films across this range. Atomic force microscopy, for instance, requires direct access to the film, and can be slow and potentially damaging, whereas the wavenumber shift in Raman spectroscopy saturates beyond around $10 \mathrm{~nm}$. Kyoungsik Yu and colleagues at the Korea Advanced Institute of Science and Technology have now developed a method to optically measure the thicknesses of hBN films from 10 to $500 \mathrm{~nm}$.

The researchers first calculate the underlying silicon dioxide thickness from the difference in Raman intensity at two different wavelengths; a single wavelength would not give an unambiguous result due to interference effects in relatively thick films. Next, they measured two ratios - again to avoid interference - on the Raman intensity between the hBN film and the overall structure (substrate plus hBN), and between the overall structure and the substrate by itself, in order to calculate the thickness of the hBN. They measured $47 \mathrm{hBN}$ flakes with this technique and compared the results with measurements made using atomic force microscopy, finding an estimation error of $2.5 \%$.

Matthew Parker

Published online: 20 March 2020

https://doi.org/10.1038/s41928-020-0393-0 